\title{
Spectral analysis of the spin-boson Hamiltonian with two bosons for arbitrary coupling and bounded dispersion relation
}

\author{
Orif O. Ibrogimov \\ Department of Mathematics, Faculty of Nuclear Sciences and Physical Engineering, Czech Technical University, \\ Trojanova 13, 12000 Prague 2, Czech Republic; ibrogori@fjfi.cvut.cz
}

29 January 2019

\begin{abstract}
We study the spectrum of the spin-boson Hamiltonian with two bosons for arbitrary coupling $\alpha>0$ in the case when the dispersion relation of the free field is a bounded function. We derive an explicit description of the essential spectrum which consists of the so-called two and three-particle branches that can be separated by a gap if the coupling is sufficiently large. It turns out, that depending on the location of the coupling constant and the energy level of the atom (with respect to certain constants depending on the maximal and the minimal values of the boson energy) as well as the validity or the violation of the infrared regularity type conditions, the essential spectrum is either a single finite interval or a disjoint union of at most six finite intervals. The corresponding critical values of the coupling constant are determined explicitly and the asymptotic lengths of the possible gaps are given when $\alpha$ approaches to the respective critical value. Under minimal smoothness and regularity conditions on the boson dispersion relation and the coupling function, we show that discrete eigenvalues can never accumulate at the edges of the two-particle branch. Moreover, we show the absence of the discrete eigenvalue accumulation at the edges of the three-particle branch in the infrared regular case.
\end{abstract}

\section{Introduction}

The spin-boson Hamiltonian is the energy operator of a quantum mechanical model describing a two-level system which is linearly coupled to the quantized field of bosons. It is formally given by the expression

$$
H=\varepsilon \sigma_{z} \otimes \mathbb{I}+\mathbb{I} \otimes \int_{\mathbb{R}^{d}} \omega(k) a^{*}(k) a(k) \mathrm{d} k+\alpha \sigma_{x} \otimes \int_{\mathbb{R}^{d}}\left(\lambda(k) a^{*}(k)+\overline{\lambda(k)} a(k)\right) \mathrm{d} k
$$

and acts on the Hilbert space

$$
\mathscr{H}=\mathbb{C}^{2} \otimes \mathcal{F}_{s}\left(L^{2}\left(\mathbb{R}^{d}\right)\right),
$$

where $\mathcal{F}_{s}\left(L^{2}\left(\mathbb{R}^{d}\right)\right)$ is the symmetric (bosonic) Fock space over $\mathbb{R}^{d}$. In $\mathbb{1}$ ), $a^{*}(k)$ and $a(k)$ are the boson creation and annihilation operators,

$$
\sigma_{z}=\left(\begin{array}{cc}
1 & 0 \\
0 & -1
\end{array}\right), \quad \sigma_{x}=\left(\begin{array}{ll}
0 & 1 \\
1 & 0
\end{array}\right)
$$

are the Pauli (spin) matrices, the constants $\varepsilon>0$ and $-\varepsilon$ are the energy levels of the atom corresponding to its excited and ground states, respectively, $\omega(k)$ is the boson dispersion, $\alpha>0$ is the coupling constant and $\lambda(k)$ is the coupling function.

For the photon case, spectral and scattering properties of the full spin-boson Hamiltonian as well as of its finite photon approximations have been investigated extensively and as a by-product sophisticated

Keywords. Spin-boson Hamiltonian, Fock space, discrete/essential spectrum, Schur complement, Weyl inequality.

2010 Mathematics Subject Classification. 81Q10, 47A10, 70F07, 47G10. 
techniques have been developed. The corresponding literature is enormous and we limit ourselves to citing [47, 50, 23, 36, 20, 7, 12, 44, 10, 6, 16, 22, 19, 5, 1, 21, 15, 31, 9, 13, (and the related work 38, 37, 39, 40,). In these studies, the dispersion of the free field is taken to be either the relativistic dispersion $\omega(k)=\sqrt{k^{2}+m^{2}}$ or its limiting cases $\omega(k)=|k|, \omega(k)=\frac{k^{2}}{2 m}$, and sometimes as a general unbounded and almost everywhere continuous function preserving all the features of these physical photon dispersion relations.

In general, the dispersion relation $\omega: \mathbb{R}^{d} \rightarrow[0, \infty)$ and the coupling function $\lambda: \mathbb{R}^{d} \rightarrow \mathbb{C}$ are fixed by the physics of the problem. In view of different applications of the spin-boson Hamiltonian, one likes to consider them as free parameter functions and impose only some general conditions such as

$$
\lambda \in L^{2}\left(\mathbb{R}^{d}\right), \quad \frac{\lambda}{\sqrt{\omega-\omega_{0}}} \in L^{2}\left(\mathbb{R}^{d}\right)
$$

where

$$
\omega_{0}:=\inf _{k \in \mathbb{R}^{d}} \omega(k)
$$

We note that the first condition in (3) is the minimal assumption on the coupling function from the spectral theory viewpoint as it is needed to guarantee the closability of the energy operator. To the best of our knowledge, almost every study on the spectrum of the spin-boson model in the up-to-date literature assumes at least (3) or its strengthened version where the second condition in (3) is replaced by

$$
\frac{\lambda}{\omega-\omega_{0}} \in L^{2}\left(\mathbb{R}^{d}\right)
$$

which is known as the infrared regularity condition (cf. [22, 14]). The recent work [25] studies the problem of the explicit description of the essential spectrum and the finiteness of the discrete spectrum for the spinboson model with two photons for arbitrary coupling where the only requirement on the coupling function was its square integrability, whereas the photon dispersion relation was assumed to be a continuous (almost everywhere) and unbounded function.

Motivated by solid state physics applications of the spin-boson Hamiltonian* and related finite volume approximation problems ( $c f$. [7]), in this paper we undertake the spectral analysis of the spin-boson Hamiltonian with two bosons for arbitrary coupling and bounded dispersion relation. The corresponding truncated spin-boson Hamiltonian is obtained from the full spin-boson Hamiltonian by the compression onto the subspace of two bosons which is given by the tensor product of $\mathbb{C}^{2}$ and the truncated Fock space

$$
\mathcal{F}_{s}^{2}:=\mathbb{C} \oplus L^{2}\left(\mathbb{R}^{d}\right) \oplus L_{s}^{2}\left(\mathbb{R}^{d} \times \mathbb{R}^{d}\right)
$$

Here $L_{s}^{2}\left(\mathbb{R}^{d} \times \mathbb{R}^{d}\right)$ stands for the subspace of the Hilbert space $L^{2}\left(\mathbb{R}^{d} \times \mathbb{R}^{d}\right)$ consisting of symmetric functions and equipped with the $1 / 2$-inner product of the latter. For $f=\left(f_{0}^{(\sigma)}, f_{1}^{(\sigma)}, f_{2}^{(\sigma)}\right) \in \mathbb{C}^{2} \otimes \mathcal{F}_{s}^{2}$, where $\sigma= \pm$ is a discrete variable, the Hamiltonian of our system is given by the formal expression

$$
\begin{aligned}
\left(H_{\alpha} f\right)_{0}^{(\sigma)} & =\sigma \varepsilon f_{0}^{(\sigma)}+\alpha \int_{\mathbb{R}^{d}} \lambda(q) f_{1}^{(-\sigma)}(q) \mathrm{d} q \\
\left(H_{\alpha} f\right)_{1}^{(\sigma)}(k) & =(\sigma \varepsilon+\omega(k)) f_{1}^{(\sigma)}(k)+\alpha \lambda(k) f_{0}^{(-\sigma)}+\alpha \int_{\mathbb{R}^{d}} f_{2}^{(-\sigma)}(k, q) \lambda(q) \mathrm{d} q, \\
\left(H_{\alpha} f\right)_{2}^{(\sigma)}\left(k_{1}, k_{2}\right) & =\left(\sigma \varepsilon+\omega\left(k_{1}\right)+\omega\left(k_{2}\right)\right) f_{2}^{(\sigma)}\left(k_{1}, k_{2}\right)+\alpha \lambda\left(k_{1}\right) f_{1}^{(-\sigma)}\left(k_{2}\right)+\alpha \lambda\left(k_{2}\right) f_{1}^{(-\sigma)}\left(k_{1}\right) .
\end{aligned}
$$

Throughout the paper we assume that the parameter $\varepsilon>0$ is fixed, the dispersion relation $\omega \geq 0$ is a non-constant, bounded, continuous function (although almost everywhere continuity will be enough in our analysis) and the coupling function $\lambda$ is not identically zero and $\lambda \in L^{2}\left(\mathbb{R}^{d}\right)$. If $\lambda$ is identically zero on $\mathbb{R}^{d}$, then the bosons do not couple to the atom and the description of the spectrum becomes straightforward. The case of constant dispersion relation was considered in 24] where it was possible to make the spectral analysis very explicit. The spatial dimension, $d \geq 1$, plays no particular role in our analysis and is left arbitrary. We make the notation

$$
\omega_{1}:=\sup _{k \in \mathbb{R}^{d}} \omega(k)
$$

\footnotetext{
${ }^{*}$ We note that one has $\omega(k) \equiv 1$ for the (original) Fröhlich polaron, $c f$. [18, 46]
} 
The goal of this paper is to give an explicit description of the essential spectrum, analyse its structure and study the finiteness of the discrete eigenvalues in the gaps as well as outside of the essential spectrum for all values of the coupling constant. Our strategy is based on reducing the problem to the spectral analysis of a family of self-adjoint $2 \times 2$ operator matrices

$$
\mathcal{A}:=\left(\begin{array}{ll}
A & B \\
C & D
\end{array}\right)
$$

depending on two parameters $\gamma \in \mathbb{R}$ and $\alpha>0$ which act on the Hilbert space

$$
\mathcal{H}:=L^{2}\left(\mathbb{R}^{d}\right) \oplus L_{s}^{2}\left(\mathbb{R}^{d} \times \mathbb{R}^{d}\right) .
$$

For $f \in L^{2}\left(\mathbb{R}^{d}\right)$ and $g \in L_{s}^{2}\left(\mathbb{R}^{d} \times \mathbb{R}^{d}\right)$, the operator entries of (9) are formally defined by the relations

$$
\begin{array}{ll}
(A f)(k)=(-\gamma+\omega(k)) f(k), & (B g)(k)=\alpha \int g(k, q) \lambda(q) \mathrm{d} q, \\
(C f)(k, q)=\alpha \overline{\lambda(k)} f(q)+\alpha \overline{\lambda(q)} f(k), & (D g)(k, q)=(\gamma+\omega(k)+\omega(q)) g(k, q) .
\end{array}
$$

The methods we employ to achieve the aforementioned results are direct and quite simple. We particularly benefit from block operator matrix techniques involving Schur complements and the corresponding FrobeniusSchur factorizations combined with the standard perturbation theory. It turns out that the essential spectrum of the reduced operator matrix $\mathcal{A}$ consists of the union of the interval $\left[2 \omega_{0}+\gamma, 2 \omega_{1}+\gamma\right]$ (i.e. the spectrum of $D$ ) with an additional branch corresponding to the essential spectrum of a non-linear pencil of multiplication operators in $L^{2}\left(\mathbb{R}^{d}\right)$ which represents the Schur complement of $D-z$ in the operator matrix $\mathcal{A}-z$ in the Calkin algebra (Theorem 2.1). Depending on the location of the parameters $\alpha>0$ and $\varepsilon$ (with respect to certain constants formed out of $\omega_{0}$ and $\omega_{1}$ ) as well as the validity or the violation of the second condition in (3) and its natural counterpart

$$
\frac{\lambda}{\sqrt{\omega_{1}-\omega}} \in L^{2}\left(\mathbb{R}^{d}\right)
$$

this additional branch consists of a single finite interval which can be in either side of $\left[2 \omega_{0}+\gamma, 2 \omega_{1}+\gamma\right]$, or two finite intervals, one in each side of the latter. The corresponding critical values of the coupling constant, the transitions at which open a new gap in the essential spectrum, are found explicitly and the asymptotic lengths of the possible gaps are determined when $\alpha$ approaches to the respective critical value (see Theorem 2.2). Inspired by the splitting trick we have developed in the recent work [25], it is shown that no edge of the essential spectrum other than $2 \omega_{0}+\gamma$ and $2 \omega_{1}+\gamma$ can be an accumulation point of the discrete spectrum (Theorem [2.3). The absence of the discrete eigenvalue accumulation at the points $2 \omega_{0}+\gamma$ and $2 \omega_{1}+\gamma$ can also be guaranteed whenever the infrared regularity condition (85) and its natural counterpart

$$
\frac{\lambda}{\omega_{1}-\omega} \in L^{2}\left(\mathbb{R}^{d}\right)
$$

(or their weaker version, in the case when these points are not the top or the bottom of the essential spectrum) are satisfied (Theorem [2.4). Finally, in Theorem 3.1, we deduce the spectral properties of the Hamiltonian (7) from the spectral information for the operator matrix $\mathcal{A}$ with $\gamma=\varepsilon$ and $\gamma=-\varepsilon$.

In spite of being self-adjoint and bounded, the operator matrix $\mathcal{A}$ in (11) is, up to our knowledge, not covered by any of the currently existing abstract results such as [8, 3, 35, 32, 34, and requires an individual analysis. This is mainly due to the non-compactness of partial-integral operators and the fact that both diagonal entries have empty discrete spectrum. Although in our setting the underlying domain is the whole Euclidean space $\mathbb{R}^{d}$, our results can be trivially translated into the setting with bounded underlying domains $\Omega \subset \mathbb{R}^{d}$ such as the torus $\mathbb{T}^{d}$. To achieve this, it suffices to replace the parameter functions $\omega$ and $\lambda$ with their cut-offs $\chi_{\Omega} \omega$ and $\chi_{\Omega} \lambda$. In this sense our results generalize and clarify the main results of [40, 42] in several respects. The splitting trick that we employ to show the absence of the eigenvalue accumulation at the edges of the two-particle branch (Theorem 2.3) is a modification of the similar trick developed in our recent work [25]. Though inspired by it, our splitting trick is quite different from the well known splitting trick of Schrödinger operator theory (cf. 43, 30, 41, 17, 2, 27, ). It is based on a "special decomposition" of a "special portion" of the Birman-Schwinger type kernel. Unlike our approach, the classical splitting trick in our setting would require some additional regularity on the parameter functions. 
Throughout the paper we adopt the following notations. For a self-adjoint operator $T$ acting in a Hilbert space and an interval $(a, b) \subset \mathbb{R} \backslash \sigma_{\text {ess }}(T)$, we denote by $N((a, b) ; T)$ the dimension of the spectral subspace of $T$ corresponding to the interval $(a, b)$ which coincides with the number of the discrete eigenvalues of $T$ in the interval $(a, b)$ (counted with multiplicities). The integrals with no indication of the limits imply the integration over the whole space $\mathbb{R}^{d}$ or $\mathbb{R}^{d} \times \mathbb{R}^{d}$.

\section{Spectral properties of the reduced operator matrix}

\subsection{Preliminaries}

Since $\lambda \in L^{2}\left(\mathbb{R}^{d}\right)$, it follows that $B: L_{s}^{2}\left(\mathbb{R}^{d} \times \mathbb{R}^{d}\right) \rightarrow L^{2}\left(\mathbb{R}^{d}\right)$ is an everywhere defined bounded operator with the adjoint $B^{*}=C$. Hence, the operator matrix $\mathcal{A}: \mathcal{H} \rightarrow \mathcal{H}$ is everywhere defined and self-adjoint (cf. [29. Theorem V.4.3]). Note that $D: L_{s}^{2}\left(\mathbb{R}^{d} \times \mathbb{R}^{d}\right) \rightarrow L_{s}^{2}\left(\mathbb{R}^{d} \times \mathbb{R}^{d}\right)$ is an everywhere defined self-adjoint operator with the spectrum

$$
\sigma(D)=\left[2 \omega_{0}+\gamma, 2 \omega_{1}+\gamma\right]
$$

where $\omega_{0}$ and $\omega_{1}$ are defined by (4) and (8).

As it was mentioned in Introduction, our approach to study the spectrum of the $2 \times 2$ operator matrix $\mathcal{A}$ is based on the so-called Schur complements and the corresponding Frobenius-Schur factorization, $c f$. [49]. For $z \in \rho(D)$, the Schur complement of $D-z$ in the operator matrix $\mathcal{A}-z$ is given by

$$
S(z):=A-z-B(D-z)^{-1} B^{*}, \quad \operatorname{Dom}(S(z)):=L^{2}(\mathbb{R}) .
$$

Simple calculations yield the representation

$$
S(z)=\Delta(z)-K(z)
$$

where $\Delta(z): L^{2}\left(\mathbb{R}^{d}\right) \rightarrow L^{2}\left(\mathbb{R}^{d}\right)$ is the maximal operator of multiplication by the function

$$
\Delta(k ; z):=\omega(k)-\gamma-z-\alpha^{2} \int \frac{|\lambda(q)|^{2} \mathrm{~d} q}{\omega(k)+\omega(q)+\gamma-z},
$$

and $K(z): L^{2}\left(\mathbb{R}^{d}\right) \rightarrow L^{2}\left(\mathbb{R}^{d}\right)$ is everywhere defined integral operator with the kernel

$$
p(k, q ; z):=\frac{\alpha^{2} \overline{\lambda(k)} \lambda(q)}{\omega(k)+\omega(q)+\gamma-z} .
$$

Since $B(D-z)^{-1}: L_{s}^{2}\left(\mathbb{R}^{d} \times \mathbb{R}^{d}\right) \rightarrow L^{2}\left(\mathbb{R}^{d}\right)$ is an everywhere defined bounded operator, the following Frobenius-Schur factorization holds

$$
\mathcal{A}-z=\left(\begin{array}{cc}
I & B(D-z)^{-1} \\
0 & I
\end{array}\right)\left(\begin{array}{cc}
S(z) & 0 \\
0 & D-z
\end{array}\right)\left(\begin{array}{cc}
I & 0 \\
(D-z)^{-1} B^{*} & I
\end{array}\right) .
$$

In view this factorization, we can reduce the study of the essential spectrum of the operator matrix $\mathcal{A}$ to that of the non-linear pencil $S$. More precisely, we have

$$
\sigma_{\mathrm{ess}}(\mathcal{A}) \cap \rho(D)=\sigma_{\mathrm{ess}}(S) \cap \rho(D) .
$$

Moreover, we have the following crucial result the proof of which is similar to that of [25, Lemma 3] and follows from the factorization (18).

Lemma 2.1. If $z \in \rho(D)$ satisfies $N((-\infty, 0) ; S(z))<\infty$, then there exists $\delta>0$ such that

$$
\sigma_{\operatorname{disc}}(\mathcal{A}) \cap(z-\delta, z)=\varnothing
$$

Similarly, if $z \in \rho(D)$ satisfies $N((-\infty, 0) ;-S(z))<\infty$, then there exists $\delta>0$ such that

$$
\sigma_{\text {disc }}(\mathcal{A}) \cap(z, z+\delta)=\varnothing .
$$


Remark 2.1. For each $z \in \rho(D)$, the operators $S(z): L^{2}\left(\mathbb{R}^{d}\right) \rightarrow L^{2}\left(\mathbb{R}^{d}\right)$ and $\Delta(z): L^{2}\left(\mathbb{R}^{d}\right) \rightarrow L^{2}\left(\mathbb{R}^{d}\right)$ are bounded and self-adjoint on $\mathcal{H}_{1}$. Moreover, the integral operator $K(z): L^{2}\left(\mathbb{R}^{d}\right) \rightarrow L^{2}\left(\mathbb{R}^{d}\right)$ is self-adjoint and of Hilbert-Schmidt class since its kernel $p(\cdot, \cdot ; z)$ satisfies

$$
\|p(\cdot, \cdot ; z)\|_{L_{s}^{2}\left(\mathbb{R}^{d} \times \mathbb{R}^{d}\right)} \leq \frac{2 \alpha^{2}\|\lambda\|_{L^{2}\left(\mathbb{R}^{d}\right)}^{2}}{\operatorname{dist}(z, \sigma(D))}<\infty .
$$

The rest of this subsection is devoted to the analysis of possible zeros of the continuous function

$$
\Phi(z)=-\gamma-z-\alpha^{2} \int \frac{|\lambda(q)|^{2} \mathrm{~d} q}{\omega(q)+\gamma-z}, \quad z \in\left(-\infty, \omega_{0}+\gamma\right) \cup\left(\omega_{1}+\gamma, \infty\right) .
$$

It is easy to see that $\Phi$ is strictly decreasing and

$$
\lim _{z \downarrow-\infty} \Phi(z)=+\infty, \quad \lim _{z \uparrow+\infty} \Phi(z)=-\infty .
$$

Moreover, the monotone convergence theorem guarantees the existence of the (possibly improper) limits

$$
\lim _{z \uparrow \omega_{0}+\gamma} \Phi(z)=: \Phi\left(\omega_{0}+\gamma\right), \quad \lim _{z \downarrow \omega_{1}+\gamma} \Phi(z)=: \Phi\left(\omega_{1}+\gamma\right) .
$$

If the condition

$$
\frac{\lambda}{\sqrt{\omega-\omega_{0}}} \in L^{2}\left(\mathbb{R}^{d}\right)
$$

is satisfied, then the first limit in (25) is finite for all $\alpha>0$ and is given by

$$
\Phi\left(\omega_{0}+\gamma\right):=-2 \gamma-\omega_{0}-\alpha^{2} \int \frac{|\lambda(q)|^{2} \mathrm{~d} q}{\omega(q)-\omega_{0}} .
$$

We distinguish the two cases:

- Either $\gamma \geq-\frac{\omega_{0}}{2}$ and $\alpha>0$ is arbitrary, or $\gamma<-\frac{\omega_{0}}{2}$ and $\alpha>\alpha_{1}$, where

$$
\alpha_{1}:=\alpha_{1}(\gamma):=\frac{\sqrt{-2 \gamma-\omega_{0}}}{\left\|\frac{\lambda}{\sqrt{\omega-\omega_{0}}}\right\|_{L^{2}\left(\mathbb{R}^{d}\right)}}
$$

In this case, we have $\Phi\left(\omega_{0}+\gamma\right)<0$. Hence, the first relation in (24) and the monotonicity imply that the continuous function $\Phi$ has a unique zero - denoted by $E:=E(\alpha, \gamma)$ - in the interval $\left(-\infty, \omega_{0}+\gamma\right)$.

- The case $\gamma<-\frac{\omega_{0}}{2}$ and $0<\alpha \leq \alpha_{1}$. In this case, we have $\Phi\left(\omega_{0}+\gamma\right) \geq 0$. Hence, the first relation in (24) and the monotonicity imply that the continuous function $\Phi$ does not vanish in the interval $\left(-\infty, \omega_{0}+\gamma\right)$.

If (26) does not hold, then the first limit in (25) is negative infinity for all $\alpha>0$. Hence, the first relation in (24) and the monotonicity imply that the continuous function $\Phi$ has a unique zero (again denoted by) $E:=E(\gamma, \alpha)$ in the interval $\left(-\infty, \omega_{0}+\gamma\right)$ for all $\alpha>0$.

Similarly, if the condition

$$
\frac{\lambda}{\sqrt{\omega_{1}-\omega}} \in L^{2}\left(\mathbb{R}^{d}\right)
$$

is satisfied, then the second limit in (25) is finite for all $\alpha>0$ and is given by

$$
\Phi\left(\omega_{1}+\gamma\right):=-2 \gamma-\omega_{1}+\alpha^{2} \int \frac{|\lambda(q)|^{2} \mathrm{~d} q}{\omega_{1}-\omega(q)} .
$$

We again distinguish the two cases:

- Either $\gamma \leq-\frac{\omega_{1}}{2}$ and $\alpha>0$ is arbitrary, or $\gamma>-\frac{\omega_{1}}{2}$ and $\alpha>\alpha_{3}$, where

$$
\alpha_{3}:=\alpha_{3}(\gamma):=\frac{\sqrt{\omega_{1}+2 \gamma}}{\left\|\frac{\lambda}{\sqrt{\omega_{1}-\omega}}\right\|_{L^{2}\left(\mathbb{R}^{d}\right)} .}
$$

In this case, we have $\Phi\left(\omega_{1}+\gamma\right)>0$. Hence, the second relation in (24) and the monotonicity imply that the continuous function $\Phi$ has a unique zero - denoted by $F:=F(\gamma, \alpha)$ - in the interval $\left(\omega_{1}+\gamma, \infty\right)$. 
- The case $\gamma>-\frac{\omega_{1}}{2}$ and $0<\alpha \leq \alpha_{3}$. In this case, we have $\Phi\left(\omega_{1}+\gamma\right) \leq 0$. Hence, the second relation in (24) and the monotonicity imply that the continuous function $\Phi$ does not vanish in the interval $\left(\omega_{1}+\gamma, \infty\right)$.

If (29) does not hold, then the second limit in (25) is positive infinity for all $\alpha>0$. Hence, the second relation in (24) and the monotonicity imply that the continuous function $\Phi$ has a unique zero (again denoted by) $F:=F(\gamma, \alpha)$ in the interval $\left(\omega_{1}+\gamma, \infty\right)$ for all $\alpha>0$.

In addition to (28) and (31), we will also be dealing with the following particular values of the coupling constant

$$
\alpha_{2}:=\alpha_{2}(\gamma):=\frac{\sqrt{\omega_{1}-2 \omega_{0}-2 \gamma}}{\left\|\frac{\lambda}{\sqrt{\omega_{1}-2 \omega_{0}+\omega}}\right\|_{L^{2}\left(\mathbb{R}^{d}\right)}}, \quad \alpha_{4}:=\alpha_{4}(\gamma):=\frac{\sqrt{2 \omega_{1}-\omega_{0}+2 \gamma}}{\left\|\frac{\lambda}{\sqrt{2 \omega_{1}-\omega_{0}-\omega}}\right\|_{L^{2}\left(\mathbb{R}^{d}\right)}}
$$

whenever $\gamma \leq \frac{\omega_{1}}{2}-\omega_{0}$ and $\gamma \geq \frac{\omega_{0}}{2}-\omega_{1}$, respectively. Since the denominators of both fractions in (32) lie between $\frac{1}{\sqrt{2\left(\omega_{1}-\omega_{0}\right)}}\|\lambda\|_{L^{2}\left(\mathbb{R}^{d}\right)}$ and $\frac{1}{\sqrt{\omega_{1}-\omega_{0}}}\|\lambda\|_{L^{2}\left(\mathbb{R}^{d}\right)}$, the quantities in (32) are well-defined.

\subsection{Essential spectrum of the reduced operator matrix}

The following theorem provides an explicit description of the essential spectrum of the self-adjoint operator matrix $\mathcal{A}$ corresponding to (9)-(11). The structure of the essential spectrum depends on the location of the parameters $\alpha>0$ and $\gamma \in \mathbb{R}$ as well as the violation of the conditions (26) and (29).

Theorem 2.1. Let the coupling constant $\alpha>0$ be arbitrary.

(i) Suppose that both of (26) and (29) are violated.

(a) If $\gamma \in\left(-\infty, \frac{\omega_{0}}{2}-\omega_{1}\right]$, then

$$
\sigma_{\mathrm{ess}}(\mathcal{A})= \begin{cases}{\left[\omega_{0}+E, 2 \omega_{1}+\gamma\right] \cup\left[\omega_{0}+F, \omega_{1}+F\right]} & \text { if } \alpha \in\left(0, \alpha_{2}\right], \\ {\left[\omega_{0}+E, \omega_{1}+E\right] \cup\left[2 \omega_{0}+\gamma, 2 \omega_{1}+\gamma\right] \cup\left[\omega_{0}+F, \omega_{1}+F\right]} & \text { if } \alpha \in\left(\alpha_{2}, \infty\right) .\end{cases}
$$

(b) If $\gamma \in\left(\frac{\omega_{0}}{2}-\omega_{1}, \frac{\omega_{1}}{2}-\omega_{0}\right]$, then

$$
\sigma_{\mathrm{ess}}(\mathcal{A})= \begin{cases}{\left[\omega_{0}+E, \omega_{1}+F\right]} & \text { if } \alpha \in\left(0, \alpha_{2}\right], \\ {\left[\omega_{0}+E, \omega_{1}+E\right] \cup\left[2 \omega_{0}+\gamma, \omega_{1}+F\right]} & \text { if } \alpha \in\left(\alpha_{2}, \alpha_{4}\right], \\ {\left[\omega_{0}+E, \omega_{1}+E\right] \cup\left[2 \omega_{0}+\gamma, 2 \omega_{1}+\gamma\right] \cup\left[\omega_{0}+F, \omega_{1}+F\right]} & \text { if } \alpha \in\left(\alpha_{4}, \infty\right) .\end{cases}
$$

(c) If $\gamma \in\left(\frac{\omega_{1}}{2}-\omega_{0}, \infty\right)$, then

$$
\sigma_{\mathrm{ess}}(\mathcal{A})= \begin{cases}{\left[\omega_{0}+E, \omega_{1}+E\right] \cup\left[2 \omega_{0}+\gamma, \omega_{1}+F\right]} & \text { if } \alpha \in\left(0, \alpha_{4}\right], \\ {\left[\omega_{0}+E, \omega_{1}+E\right] \cup\left[2 \omega_{0}+\gamma, 2 \omega_{1}+\gamma\right] \cup\left[\omega_{0}+F, \omega_{1}+F\right]} & \text { if } \alpha \in\left(\alpha_{4}, \infty\right) .\end{cases}
$$

(ii) Suppose that (26) is satisfied but (29) is violated.

(a) If $\gamma \in\left(-\infty, \frac{\omega_{0}}{2}-\omega_{1}\right]$, then

$$
\sigma_{\mathrm{ess}}(\mathcal{A})= \begin{cases}{\left[2 \omega_{0}+\gamma, 2 \omega_{1}+\gamma\right] \cup\left[\omega_{0}+F, \omega_{1}+F\right]} & \text { if } \alpha \in\left(0, \alpha_{1}\right], \\ {\left[\omega_{0}+E, 2 \omega_{1}+\gamma\right] \cup\left[\omega_{0}+F, \omega_{1}+F\right]} & \text { if } \alpha \in\left(\alpha_{1}, \alpha_{2}\right], \\ {\left[\omega_{0}+E, \omega_{1}+E\right] \cup\left[2 \omega_{0}+\gamma, 2 \omega_{1}+\gamma\right] \cup\left[\omega_{0}+F, \omega_{1}+F\right]} & \text { if } \alpha \in\left(\alpha_{2}, \infty\right) .\end{cases}
$$

(b) If $\gamma \in\left(\frac{\omega_{0}}{2}-\omega_{1},-\frac{\omega_{0}}{2}\right]$, then

$$
\sigma_{\mathrm{ess}}(\mathcal{A})=\left\{\begin{array}{lr}
{\left[2 \omega_{0}+\gamma, \omega_{1}+F\right]} & \text { if } \alpha \in\left(0, \alpha_{1}\right], \\
{\left[\omega_{0}+E, \omega_{1}+F\right]} & \text { if } \alpha \in\left(\alpha_{1}, \alpha_{2}\right], \\
{\left[\omega_{0}+E, \omega_{1}+E\right] \cup\left[2 \omega_{0}+\gamma, \omega_{1}+F\right]} & \text { if } \alpha \in\left(\alpha_{2}, \alpha_{4}\right], \\
{\left[\omega_{0}+E, \omega_{1}+E\right] \cup\left[2 \omega_{0}+\gamma, 2 \omega_{1}+\gamma\right] \cup\left[\omega_{0}+F, \omega_{1}+F\right]} & \text { if } \alpha \in\left(\alpha_{4}, \infty\right) .
\end{array}\right.
$$


(c) If $\gamma \in\left(-\frac{\omega_{0}}{2}, \frac{\omega_{1}}{2}-\omega_{0}\right]$, then

$$
\sigma_{\mathrm{ess}}(\mathcal{A})= \begin{cases}{\left[\omega_{0}+E, \omega_{1}+F\right]} & \text { if } \alpha \in\left(0, \alpha_{2}\right], \\ {\left[\omega_{0}+E, \omega_{1}+E\right] \cup\left[2 \omega_{0}+\gamma, \omega_{1}+F\right]} & \text { if } \alpha \in\left(\alpha_{2}, \alpha_{4}\right], \\ {\left[\omega_{0}+E, \omega_{1}+E\right] \cup\left[2 \omega_{0}+\gamma, 2 \omega_{1}+\gamma\right] \cup\left[\omega_{0}+F, \omega_{1}+F\right]} & \text { if } \alpha \in\left(\alpha_{4}, \infty\right) .\end{cases}
$$

(d) If $\gamma \in\left(\frac{\omega_{1}}{2}-\omega_{0}, \infty\right)$, then

$$
\sigma_{\text {ess }}(\mathcal{A})= \begin{cases}{\left[\omega_{0}+E, \omega_{1}+E\right] \cup\left[2 \omega_{0}+\gamma, \omega_{1}+F\right]} & \text { if } \alpha \in\left(0, \alpha_{4}\right], \\ {\left[\omega_{0}+E, \omega_{1}+E\right] \cup\left[2 \omega_{0}+\gamma, 2 \omega_{1}+\gamma\right] \cup\left[\omega_{0}+F, \omega_{1}+F\right]} & \text { if } \alpha \in\left(\alpha_{4}, \infty\right) .\end{cases}
$$

(iii) Suppose that (26) is violated but (29) is satisfied.

(a) If $\gamma \in\left(-\infty, \frac{\omega_{0}}{2}-\omega_{1}\right]$, then

$$
\sigma_{\text {ess }}(\mathcal{A})= \begin{cases}{\left[\omega_{0}+E, 2 \omega_{1}+\gamma\right] \cup\left[\omega_{0}+F, \omega_{1}+F\right]} & \text { if } \alpha \in\left(0, \alpha_{2}\right], \\ {\left[\omega_{0}+E, \omega_{1}+E\right] \cup\left[2 \omega_{0}+\gamma, 2 \omega_{1}+\gamma\right] \cup\left[\omega_{0}+F, \omega_{1}+F\right]} & \text { if } \alpha \in\left(\alpha_{2}, \infty\right) .\end{cases}
$$

(b) If $\gamma \in\left(\frac{\omega_{0}}{2}-\omega_{1},-\frac{\omega_{1}}{2}\right]$, then

$$
\sigma_{\mathrm{ess}}(\mathcal{A})= \begin{cases}{\left[\omega_{0}+E, \omega_{1}+F\right]} & \text { if } \alpha \in\left(0, \alpha_{2}\right], \\ {\left[\omega_{0}+E, \omega_{1}+E\right] \cup\left[2 \omega_{0}+\gamma, \omega_{1}+F\right]} & \text { if } \alpha \in\left(\alpha_{2}, \alpha_{4}\right], \\ {\left[\omega_{0}+E, \omega_{1}+E\right] \cup\left[2 \omega_{0}+\gamma, 2 M+\gamma\right] \cup\left[\omega_{0}+F, \omega_{1}+F\right]} & \text { if } \alpha \in\left(\alpha_{4}, \infty\right) .\end{cases}
$$

(c) If $\gamma \in\left(-\frac{\omega_{1}}{2}, \frac{\omega_{1}}{2}-\omega_{0}\right]$, then

$$
\sigma_{\mathrm{ess}}(\mathcal{A})= \begin{cases}{\left[\omega_{0}+E, 2 \omega_{1}+\gamma\right]} & \text { if } \alpha \in\left(0, \alpha_{2}\right], \\ {\left[\omega_{0}+E, \omega_{1}+E\right] \cup\left[2 \omega_{0}+\gamma, 2 \omega_{1}+\gamma\right]} & \text { if } \alpha \in\left(\alpha_{2}, \alpha_{3}\right], \\ {\left[\omega_{0}+E, \omega_{1}+E\right] \cup\left[2 \omega_{0}+\gamma, \omega_{1}+F\right]} & \text { if } \alpha \in\left(\alpha_{3}, \alpha_{4}\right], \\ {\left[\omega_{0}+E, \omega_{1}+E\right] \cup\left[2 \omega_{0}+\gamma, 2 \omega_{1}+\gamma\right] \cup\left[\omega_{0}+F, \omega_{1}+F\right]} & \text { if } \alpha \in\left(\alpha_{4}, \infty\right) .\end{cases}
$$

(d) If $\gamma \in\left(\frac{\omega_{1}}{2}-\omega_{0}, \infty\right)$, then

$$
\sigma_{\text {ess }}(\mathcal{A})= \begin{cases}{\left[\omega_{0}+E, \omega_{1}+E\right] \cup\left[2 \omega_{0}+\gamma, 2 \omega_{1}+\gamma\right]} & \text { if } \alpha \in\left(0, \alpha_{3}\right], \\ {\left[\omega_{0}+E, \omega_{1}+E\right] \cup\left[2 \omega_{0}+\gamma, \omega_{1}+F\right]} & \text { if } \alpha \in\left(\alpha_{3}, \alpha_{4}\right], \\ {\left[\omega_{0}+E, \omega_{1}+E\right] \cup\left[2 \omega_{0}+\gamma, 2 \omega_{1}+\gamma\right] \cup\left[\omega_{0}+F, \omega_{1}+F\right]} & \text { if } \alpha \in\left(\alpha_{4}, \infty\right) .\end{cases}
$$

(iv) Suppose that both of (26) and (29) are satisfied.

(a) If $\gamma \in\left(-\infty, \frac{\omega_{0}}{2}-\omega_{1}\right]$, then

$$
\sigma_{\mathrm{ess}}(\mathcal{A})= \begin{cases}{\left[2 \omega_{0}+\gamma, 2 \omega_{1}+\gamma\right] \cup\left[\omega_{0}+F, \omega_{1}+F\right]} & \text { if } \alpha \in\left(0, \alpha_{1}\right], \\ {\left[\omega_{0}+E, 2 \omega_{1}+\gamma\right] \cup\left[\omega_{0}+F, \omega_{1}+F\right]} & \text { if } \alpha \in\left(\alpha_{1}, \alpha_{2}\right], \\ {\left[\omega_{0}+E, \omega_{1}+E\right] \cup\left[2 \omega_{0}+\gamma, 2 \omega_{1}+\gamma\right] \cup\left[\omega_{0}+F, \omega_{1}+F\right]} & \text { if } \alpha \in\left(\alpha_{2}, \infty\right) .\end{cases}
$$

(b) If $\gamma \in\left(\frac{\omega_{0}}{2}-\omega_{1},-\frac{\omega_{1}}{2}\right]$, then

$$
\sigma_{\mathrm{ess}}(\mathcal{A})= \begin{cases}{\left[2 \omega_{0}+\gamma, \omega_{1}+F\right]} & \text { if } \alpha \in\left(0, \alpha_{1}\right], \\ {\left[\omega_{0}+E, \omega_{1}+F\right]} & \text { if } \alpha \in\left(\alpha_{1}, \alpha_{2}\right], \\ {\left[\omega_{0}+E, \omega_{1}+E\right] \cup\left[2 \omega_{0}+\gamma, \omega_{1}+F\right]} & \text { if } \alpha \in\left(\alpha_{2}, \alpha_{4}\right], \\ {\left[\omega_{0}+E, \omega_{1}+E\right] \cup\left[2 \omega_{0}+\gamma, 2 \omega_{1}+\gamma\right] \cup\left[\omega_{0}+F, \omega_{1}+F\right]} & \text { if } \alpha \in\left(\alpha_{4}, \infty\right) .\end{cases}
$$


(c) If $\gamma \in\left(-\frac{\omega_{1}}{2},-\frac{\omega_{0}}{2}\right]$, then

$$
\sigma_{\mathrm{ess}}(\mathcal{A})= \begin{cases}{\left[2 \omega_{0}+\gamma, 2 \omega_{1}+\gamma\right]} & \text { if } \alpha \in\left(0, \alpha_{1}\right], \\ {\left[\omega_{0}+E, 2 \omega_{1}+\gamma\right]} & \text { if } \alpha \in\left(\alpha_{1}, \alpha_{2}\right], \\ {\left[\omega_{0}+E, \omega_{1}+E\right] \cup\left[2 \omega_{0}+\gamma, 2 \omega_{1}+\gamma\right]} & \text { if } \alpha \in\left(\alpha_{2}, \alpha_{3}\right], \\ {\left[\omega_{0}+E, \omega_{1}+E\right] \cup\left[2 \omega_{0}+\gamma, \omega_{1}+F\right]} & \text { if } \alpha \in\left(\alpha_{3}, \alpha_{4}\right], \\ {\left[\omega_{0}+E, \omega_{1}+E\right] \cup\left[2 \omega_{0}+\gamma, 2 \omega_{1}+\gamma\right] \cup\left[\omega_{0}+F, \omega_{1}+F\right]} & \text { if } \alpha \in\left(\alpha_{4}, \infty\right) .\end{cases}
$$

(d) If $\gamma \in\left(-\frac{\omega_{0}}{2}, \frac{\omega_{1}}{2}-\omega_{0}\right]$, then

$$
\sigma_{\mathrm{ess}}(\mathcal{A})= \begin{cases}{\left[\omega_{0}+E, 2 \omega_{1}+\gamma\right]} & \text { if } \alpha \in\left(0, \alpha_{2}\right], \\ {\left[\omega_{0}+E, \omega_{1}+E\right] \cup\left[2 \omega_{0}+\gamma, 2 \omega_{1}+\gamma\right]} & \text { if } \alpha \in\left(\alpha_{2}, \alpha_{3}\right], \\ {\left[\omega_{0}+E, \omega_{1}+E\right] \cup\left[2 \omega_{0}+\gamma, \omega_{1}+F\right]} & \text { if } \alpha \in\left(\alpha_{3}, \alpha_{4}\right], \\ {\left[\omega_{0}+E, \omega_{1}+E\right] \cup\left[2 \omega_{0}+\gamma, 2 \omega_{1}+\gamma\right] \cup\left[\omega_{0}+F, \omega_{1}+F\right]} & \text { if } \alpha \in\left(\alpha_{4}, \infty\right) .\end{cases}
$$

(e) If $\gamma \in\left(\frac{\omega_{1}}{2}-\omega_{0}, \infty\right)$, then

$$
\sigma_{\mathrm{ess}}(\mathcal{A})= \begin{cases}{\left[\omega_{0}+E, \omega_{1}+E\right] \cup\left[2 \omega_{0}+\gamma, 2 \omega_{1}+\gamma\right]} & \text { if } \alpha \in\left(0, \alpha_{3}\right], \\ {\left[\omega_{0}+E, \omega_{1}+E\right] \cup\left[2 \omega_{0}+\gamma, \omega_{1}+F\right]} & \text { if } \alpha \in\left(\alpha_{3}, \alpha_{4}\right], \\ {\left[\omega_{0}+E, \omega_{1}+E\right] \cup\left[2 \omega_{0}+\gamma, 2 \omega_{1}+\gamma\right] \cup\left[\omega_{0}+F, \omega_{1}+F\right]} & \text { if } \alpha \in\left(\alpha_{4}, \infty\right) .\end{cases}
$$

Proof. Let $z_{0} \in\left(2 \omega_{0}+\gamma, 2 \omega_{1}+\gamma\right)$ be arbitrary. Then $z_{0}=2 \omega\left(k_{0}\right)+\gamma$ for some $k_{0} \in \mathbb{R}^{d}$. Further, let $\varphi \in C^{\infty}\left(\mathbb{R}^{d}\right)$ be an arbitrary function supported in the annulus $\left\{k \in \mathbb{R}^{d}: 0.5 \leq\|k\|<1\right\}$ and such that $\|\varphi\|_{L^{2}\left(\mathbb{R}^{d}\right)}=1$. It follows that the orthonormal system $\left\{\Psi_{n}\right\}_{n \in \mathbb{N}}:=\left\{\left(0, \psi_{n}\right)^{t}\right\}_{n \in \mathbb{N}}$, where

$$
\psi_{n}(k, q):=2^{n d+\frac{1}{2}} \varphi\left(2^{n}\left(k-k_{0}\right)\right) \varphi\left(2^{n}\left(q-k_{0}\right)\right), \quad k, q \in \mathbb{R}^{d},
$$

is a singular sequence for the operator matrix $\mathcal{A}-z_{0}$. Hence, as in [25, we get the inclusion

$$
\left[2 \omega_{0}+\gamma, 2 \omega_{1}+\gamma\right] \subset \sigma_{\text {ess }}(\mathcal{A}) .
$$

Since $\left[2 \omega_{0}+\gamma, 2 \omega_{1}+\gamma\right]=\sigma(D)$, we can use the Frobenius-Schur factorization (18) to describe $\sigma_{\text {ess }}(\mathcal{A}) \cap \rho(D)$, see (19). In fact, as in the proof of [25, Theorem 1], a simple perturbation argument yields

$$
\sigma_{\text {ess }}(\mathcal{A})=\left[2 \omega_{0}+\gamma, 2 \omega_{1}+\gamma\right] \cup\left\{z \notin\left[2 \omega_{0}+\gamma, 2 \omega_{1}+\gamma\right]: 0 \in \sigma_{\text {ess }}(\Delta(z))\right\} .
$$

Since $\Phi$ is strictly decreasing on its domain of definition, we have

$$
\inf _{k \in \mathbb{R}^{d}} \Delta(k ; z)=\Phi\left(z-\omega_{0}\right), \quad \sup _{k \in \mathbb{R}^{d}} \Delta(k ; z)=\Phi\left(z-\omega_{1}\right)
$$

for all $z \in\left(-\infty, 2 \omega_{0}+\gamma\right) \cup\left(2 \omega_{1}+\gamma, \infty\right)$. This and the continuity of $\Delta(\cdot, \cdot)$ in the second variable imply that

$$
\sigma_{\text {ess }}(\mathcal{A})=\Sigma_{1} \cup\left[2 \omega_{0}+\gamma, 2 \omega_{1}+\gamma\right] \cup \Sigma_{2},
$$

where

$$
\Sigma_{1}:=\left\{z<2 \omega_{0}+\gamma: \Phi\left(z-\omega_{0}\right) \leq 0 \leq \Phi\left(z-\omega_{1}\right)\right\}
$$

and

$$
\Sigma_{2}:=\left\{z>2 \omega_{1}+\gamma: \Phi\left(z-\omega_{0}\right) \leq 0 \leq \Phi\left(z-\omega_{1}\right)\right\} .
$$

On the other hand, by the monotonicity of the continuous function $\Phi$, we easily obtain

$$
\Sigma_{1}= \begin{cases}\varnothing & \text { if } \Phi\left(\omega_{0}+\gamma\right) \geq 0, \\ {\left[\omega_{0}+E, 2 \omega_{0}+\gamma\right)} & \text { if } \Phi\left(\omega_{0}+\gamma\right)<0 \text { and } \Phi\left(2 \omega_{0}-\omega_{1}+\gamma\right) \geq 0, \\ {\left[\omega_{0}+E, \omega_{1}+E\right]} & \text { if } \Phi\left(2 \omega_{0}-\omega_{1}+\gamma\right)<0\end{cases}
$$


and, similarly,

$$
\Sigma_{2}= \begin{cases}\varnothing & \text { if } \Phi\left(\omega_{1}+\gamma\right) \leq 0 \\ \left(2 \omega_{1}+\gamma, \omega_{1}+F\right] & \text { if } \Phi\left(\omega_{1}+\gamma\right)>0 \text { and } \Phi\left(2 \omega_{1}-\omega_{0}+\gamma\right) \leq 0 \\ {\left[\omega_{0}+F, \omega_{1}+F\right]} & \text { if } \Phi\left(2 \omega_{1}-\omega_{0}+\gamma\right)>0\end{cases}
$$

Now elementary analyses of the signs of $\Phi\left(\omega_{0}+\gamma\right), \Phi\left(\omega_{1}+\gamma\right), \Phi\left(2 \omega_{0}-\omega_{1}+\gamma\right)$ and $\Phi\left(2 \omega_{1}-\omega_{0}+\gamma\right)$ in terms of the parameters $\alpha$ and $\gamma$ together with (37) complete the proof.

Theorem 2.2. For each fixed $\gamma \in \mathbb{R}$, the following asymptotic expansions hold

$$
\begin{aligned}
& E(\alpha)=2 \omega_{0}-\omega_{1}+\gamma-\frac{2\left(\omega_{1}-2 \omega_{0}-2 \gamma\right)}{\alpha_{2}+\alpha_{2}^{3}\left\|\frac{\lambda}{\omega+\omega_{1}-2 \omega_{0}}\right\|_{L^{2}\left(\mathbb{R}^{d}\right)}^{2}}\left(\alpha-\alpha_{2}\right)+\mathrm{o}\left(\alpha-\alpha_{2}\right), \quad \alpha \downarrow \alpha_{2}, \\
& F(\alpha)=2 \omega_{1}-\omega_{0}+\gamma+\frac{2\left(2 \omega_{1}-\omega_{0}+2 \gamma\right)}{\alpha_{4}+\alpha_{4}^{3}\left\|\frac{\lambda}{2 \omega_{1}-\omega_{0}-\omega}\right\|_{L^{2}\left(\mathbb{R}^{d}\right)}^{2}}\left(\alpha-\alpha_{4}\right)+\mathrm{o}\left(\alpha-\alpha_{4}\right), \quad \alpha \uparrow \alpha_{4},
\end{aligned}
$$

provided that $\gamma \leq \frac{\omega_{1}}{2}-\omega_{0}$ and $\gamma \geq \frac{\omega_{0}}{2}-\omega_{1}$, respectively.

Proof. First of all, we note that the quantities involved in the denominators of the second terms in the asymptotic expansions (42) are well-defined as a consequence of the simple estimate

$$
\max \left\{\left\|\frac{\lambda}{\omega+\omega_{1}-2 \omega_{0}}\right\|_{L^{2}\left(\mathbb{R}^{d}\right)},\left\|\frac{\lambda}{2 \omega_{1}-\omega_{0}-\omega}\right\|_{L^{2}\left(\mathbb{R}^{d}\right)}\right\} \leq\left(\omega_{1}-\omega_{0}\right)^{-1}\|\lambda\|_{L^{2}\left(\mathbb{R}^{d}\right)} .
$$

We derive the first asymptotic expansion only. The second one can be derived similarly. To this end, let us consider the function

$$
\psi(x, y)=-\gamma-y-x^{2} \int \frac{|\lambda(q)|^{2} \mathrm{~d} q}{\omega(q)+\gamma-y}
$$

for $(x, y) \in\left[\alpha_{2}, \infty\right) \times\left(-\infty, \omega_{0}+\gamma\right]$. We have $\psi\left(\alpha_{2}, 2 \omega_{0}-\omega_{1}+\gamma\right)=0$ and

$$
\frac{\partial \psi}{\partial y}\left(\alpha_{2}, 2 \omega_{0}-\omega_{1}+\gamma\right)=-1-\alpha_{2}^{2} \int \frac{|\lambda(q)|^{2} \mathrm{~d} q}{\left(\omega(q)-2 \omega_{0}+\omega_{1}\right)^{2}} \leq-1 .
$$

Hence, the implicit function theorem yields the existence of a constant $\delta>0$ and a unique continuously differentiable function $\widehat{E}:\left[\alpha_{2}, \alpha_{2}+\delta\right) \rightarrow \mathbb{R}$ such that

$$
\widehat{E}\left(\alpha_{2}\right)=2 \omega_{0}-\omega_{1}+\gamma
$$

and $\psi(\alpha, \widehat{E}(\alpha))=0$ for all $\alpha \in\left[\alpha_{2}, \alpha_{2}+\delta\right)$. Moreover, we have

$$
\widehat{E}^{\prime}\left(\alpha_{2}+0\right)=-\frac{2\left(\omega_{1}-2 \omega_{0}-2 \gamma\right)}{\alpha_{2}+\alpha_{2}^{3}\left\|\frac{\lambda}{\omega+\omega_{1}-2 \omega_{0}}\right\|_{L^{2}\left(\mathbb{R}^{d}\right)}^{2}} .
$$

On the other hand, $\Phi(\widehat{E}(\alpha))=0$ for all $\alpha \in\left[\alpha_{2}, \alpha_{2}+\delta\right)$ and, since $E(\alpha)$ is the unique zero of the function $\Phi$ in the interval $\left(-\infty, \omega_{0}+\gamma\right)$ for all $\alpha \geq \alpha_{2}$, we conclude that $E(\alpha)=\widehat{E}(\alpha)$ for all $\alpha \in\left[\alpha_{2}, \alpha_{2}+\delta\right)$. This implies that $E:\left[\alpha_{2}, \alpha_{2}+\delta\right) \rightarrow \mathbb{R}$ is continuously differentiable and the desired asymptotic expansion follows from (45) and (46).

\subsection{Discrete spectrum of the reduced operator matrix}

Theorem 2.3. Let the coupling constant $\alpha>0$ be arbitrary. Then none of $\omega_{0}+E, \omega_{1}+E, \omega_{0}+F$ and $\omega_{1}+F$ can be an accumulation point of the discrete spectrum of the operator matrix $\mathcal{A}$.

Proof. The proof in the case when $\omega_{0}+E$ is the bottom (resp. $\omega_{1}+F$ is the top) of the essential spectrum is completely analogous to that of [25, Theorem 1ii)]. Here we give a proof of the absence of the discrete eigenvalue accumulation at $\omega_{1}+E$ from the right only. The proof of of the absence of the discrete eigenvalue accumulation at $m+F$ from the left is analogous. To this end, let $\omega_{1}+E$ be an edge of the essential spectrum 
so that $\left(\omega_{1}+E, 2 \omega_{0}+\gamma\right)$ is an essential spectral gap (see Theorem 2.1). Let $z \in\left[\omega_{1}+E, 2 \omega_{0}+\gamma\right.$ ) be fixed for a moment. Since $\left[\omega_{1}+E, 2 \omega_{0}+\gamma\right) \subset \rho(D)$, the integral operator $K(z): L^{2}\left(\mathbb{R}^{d}\right) \rightarrow L^{2}\left(\mathbb{R}^{d}\right)$ is well-defined and compact (see Remark 2.1). Consider the decomposition

$$
\frac{1}{\omega\left(k_{1}\right)+\omega\left(k_{2}\right)+\gamma-z}=\Psi_{1}\left(k_{1}, k_{2} ; z\right)+\Psi_{2}\left(k_{1}, k_{2} ; z\right),
$$

where

$$
\Psi_{1}\left(k_{1}, k_{2} ; z\right):=\frac{1}{\omega\left(k_{1}\right)+\omega_{1}+\gamma-z}+\frac{1}{\omega\left(k_{2}\right)+\omega_{1}+\gamma-z}-\frac{1}{2 \omega_{1}+\gamma-z}
$$

and

$$
\Psi_{2}\left(k_{1}, k_{2} ; z\right):=\frac{1}{\omega\left(k_{1}\right)+\omega\left(k_{2}\right)+\gamma-z}-\Psi_{1}\left(k_{1}, k_{2} ; z\right), \quad k_{1}, k_{2} \in \mathbb{R}^{d} .
$$

Let us denote by $K_{1}(z)$ and $K_{2}(z)$ the integral operators in $L^{2}\left(\mathbb{R}^{d}\right)$ whose kernels are respectively the functions $\left(k_{1}, k_{2}\right) \mapsto \alpha^{2} \lambda\left(k_{1}\right) \overline{\lambda\left(k_{2}\right)} \Psi_{1}\left(k_{1}, k_{2} ; z\right)$ and $\left(k_{1}, k_{2}\right) \mapsto \alpha^{2} \lambda\left(k_{1}\right) \overline{\lambda\left(k_{2}\right)} \Psi_{2}\left(k_{1}, k_{2} ; z\right)$ so that we have the corresponding decomposition

$$
K(z)=K_{1}(z)+K_{2}(z) .
$$

Since each summand in (48) is uniformly bounded by the constant $\frac{1}{2 \omega_{0}+\gamma-z}$, we infer from the first condition in (3) that $K_{1}(z)$ is a well-defined rank-two operator in $L^{2}\left(\mathbb{R}^{d}\right)$. In fact, the range of $K_{1}(z)$ coincides with the subspace of $L^{2}\left(\mathbb{R}^{d}\right)$ spanned by the functions $\lambda$ and $\frac{\lambda}{\omega+\omega_{1}+\gamma-z}$. Therefore, using (15) and [11, Theorem IX.3.3], we obtain

$$
\begin{aligned}
N((-\infty, 0) ;-S(z)) & =N((-\infty, 0) ;-\Delta(z)+K(z)) \\
& =N\left((-\infty, 0) ;-\Delta(z)+K_{1}(z)+K_{2}(z)\right) \\
& \leq N\left((-\infty, 0) ;-\Delta(z)+K_{2}(z)\right)+2 .
\end{aligned}
$$

Hence, in view of Lemma 2.1, it suffices to show that

$$
N\left((-\infty, 0) ;-\Delta\left(\omega_{1}+E\right)+K_{2}\left(\omega_{1}+E\right)\right)<\infty .
$$

Let $\Omega$ denote the complement of the level set of $\omega$ corresponding to $\omega_{1}$, that is, $\Omega:=\left\{k \in \mathbb{R}^{d}: \omega(k) \neq \omega_{1}\right\}$. In view of our hypothesis on the dispersion relation $\omega$, it is clear that $\Omega$ is an open subset of $\mathbb{R}^{d}$ with positive Lebesgue measure. For $z \in\left[\omega_{1}+E, 2 \omega_{0}+\gamma\right)$, we denote by $\Delta_{\Omega}(z)$ and $K_{2, \Omega}(z)$ the restrictions of the operators $\Delta(z)$ and $K_{2}(z)$ to $L^{2}(\Omega)$, respectively. Further, we denote by $\Delta_{\Omega}(\cdot ; z)$ the restriction of the function $\Delta(\cdot ; z)$ to $\Omega$ so that $\Delta_{\Omega}(z)$ is the operator of multiplication by the function $\Delta_{\Omega}(\cdot ; z)$ in $L^{2}(\Omega)$. Since $E$ is a zero of $\Phi$, by expressing $-\Delta\left(k ; \omega_{1}+E\right)$ as $-\Delta\left(k ; \omega_{1}+E\right)+\Phi(E)$ and doing some elementary calculations, we get the identity

$$
-\Delta\left(k ; \omega_{1}+E\right)=\left(\omega_{1}-\omega(k)\right)\left(1+\alpha^{2} \int \frac{|\lambda(q)|^{2} \mathrm{~d} q}{(\omega(q)+\gamma-E)\left(\omega(k)+\omega(q)+\gamma-\omega_{1}-E\right)}\right) .
$$

This implies that

$$
-\Delta_{\Omega}\left(k ; \omega_{1}+E\right) \geq \omega_{1}-\omega(k)>0 \text { for all } k \in \Omega
$$

and that $\Delta\left(\cdot ; \omega_{1}+E\right) \equiv 0$ on the level set of $\omega$ corresponding to $\omega_{1}$. So the restriction of $\Delta\left(\omega_{1}+E\right)$ to $L^{2}\left(\mathbb{R}^{d} \backslash \Omega\right)$ is the zero operator. Moreover, it is easy to see from (48)-(49) that the restriction of $K_{2}\left(\omega_{1}+E\right)$ to $L^{2}\left(\mathbb{R}^{d} \backslash \Omega\right)$ is the zero operator, too. That is why (52) is equivalent to

$$
N\left((-\infty, 0) ;-\Delta_{\Omega}\left(\omega_{1}+E\right)+K_{2, \Omega}\left(\omega_{1}+E\right)\right)<\infty .
$$

To show the latter, first we recall the monotonicity of the function $\Phi$ in the interval $\left(\omega_{1}+E, 2 \omega_{0}+\gamma\right)$. For all $z \in\left(\omega_{1}+E, 2 \omega_{0}+\gamma\right)$, this implies that

$$
\sup _{k \in \Omega} \Delta_{\Omega}(k ; z) \leq \sup _{k \in \mathbb{R}^{d}} \Delta(k ; z)=\Phi\left(z-\omega_{1}\right)<\Phi(E)=0 .
$$


Hence, the multiplication operator $-\Delta_{\Omega}(z)$ is positive for all $z \in\left(\omega_{1}+E, 2 \omega_{0}+\gamma\right)$. Since the integral operator $K_{2, \Omega}(z): L^{2}(\Omega) \rightarrow L^{2}(\Omega)$ is well-defined and Hilbert-Schmidt, it follows that the operator

$$
T(z):=\left(-\Delta_{\Omega}(z)\right)^{-1 / 2} K_{2, \Omega}(z)\left(-\Delta_{\Omega}(z)\right)^{-1 / 2}
$$

is also well-defined and Hilbert-Schmidt for all $z \in\left(\omega_{1}+E, 2 \omega_{0}+\gamma\right)$.

Next, let us define $T\left(\omega_{1}+E\right)$ to be the integral operator in $L^{2}(\Omega)$ with the kernel

$$
\Theta\left(k_{1}, k_{2}\right):=\frac{\alpha^{2} \overline{\lambda\left(k_{1}\right)} \lambda\left(k_{2}\right) \Psi_{2}\left(k_{1}, k_{2} ; \omega_{1}+E\right)}{\sqrt{-\Delta_{\Omega}\left(k_{1} ; \omega_{1}+E\right)} \sqrt{-\Delta_{\Omega}\left(k_{2} ; \omega_{1}+E\right)}}, \quad k_{1}, k_{2} \in \Omega .
$$

Let $k_{1}, k_{2} \in \Omega$ be fixed for a moment and let $a=\omega\left(k_{1}\right)-\omega_{1}, b=\omega\left(k_{2}\right)-\omega_{1}$ and $c=\omega_{1}+\gamma-E$. Then it follows that $0<a+b+c \leq 2 c, \min \{a+c, b+c\} \geq \omega_{0}+\gamma-E, a+b+c \geq 2 \omega_{0}+\gamma-\omega_{1}-E$ and $\sqrt{a b} \leq-\frac{a+b}{2} \leq \omega_{1}-\omega_{0}$. Therefore, we get

$$
\begin{aligned}
0 \leq \Psi_{2}\left(k_{1}, k_{2} ; \omega_{1}+E\right) & =\frac{1}{a+b+c}-\frac{1}{a+c}-\frac{1}{b+c}+\frac{1}{c} \\
& =\frac{a b(a+b+2 c)}{c(a+c)(b+c)(a+b+c)} \\
& \leq \frac{\sqrt{a b}(-a-b)}{(a+c)(b+c)(a+b+c)} \\
& \leq \frac{2\left(\omega_{1}-\omega_{0}\right) \sqrt{\omega_{1}-\omega\left(k_{1}\right)} \sqrt{\omega_{1}-\omega\left(k_{2}\right)}}{\left(\omega_{0}+\gamma-E\right)^{2}\left(2 \omega_{0}+\gamma-\omega_{1}-E\right)} .
\end{aligned}
$$

Combining (58) and (53), we thus obtain the pointwise estimate

$$
\left|\Theta\left(k_{1}, k_{2}\right)\right| \leq \frac{2\left(\omega_{1}-\omega_{0}\right)\left|\lambda\left(k_{1}\right)\right|\left|\lambda\left(k_{2}\right)\right|}{\left(\omega_{0}+\gamma-E\right)^{2}\left(2 \omega_{0}+\gamma-\omega_{1}-E\right)}, \quad k_{1}, k_{2} \in \Omega
$$

Thus we infer from the first condition in (3) that $\Theta \in L^{2}(\Omega \times \Omega)$. Therefore, $T\left(\omega_{1}+E\right): L^{2}(\Omega) \rightarrow L^{2}(\Omega)$ is a Hilbert-Schmidt operator and the Lebesgue's dominated convergence theorem guarantees the rightcontinuity (with respect to the operator norm) of the operator function $T$ at $\omega_{1}+E$. This observation and the Weyl inequality (cf. [11, Chapter IX])

$$
N((-\infty,-1) ; T(z)) \leq N\left((-\infty,-0.5) ; T\left(\omega_{1}+E\right)\right)+N\left((-\infty,-0.5) ;-T\left(\omega_{1}+E\right)+T(z)\right),
$$

together with the right-continuity (with respect to the operator norm) of the operator functions $\Delta_{\Omega}$ and $K_{2, \Omega}$ yield

$$
\begin{aligned}
N\left((-\infty, 0) ;-\Delta_{\Omega}\left(\omega_{1}+E\right)+K_{2, \Omega}\left(\omega_{1}+E\right)\right) & =\lim _{z \downarrow \omega_{1}+E} N\left((-\infty, 0) ;-\Delta_{\Omega}(z)+K_{2, \Omega}(z)\right) \\
& =\lim _{z \downarrow \omega_{1}+E} N((-\infty,-1) ; T(z)) \\
& \leq N\left((-\infty,-0.5) ; T\left(\omega_{1}+E\right)\right) .
\end{aligned}
$$

However, $N\left((-\infty,-0.5) ; T\left(\omega_{1}+E\right)\right)$ must be a finite number because of the compactness of the operator $T\left(\omega_{1}+E\right)$, thus justifying (54).

Theorem 2.4. Let the coupling constant $\alpha>0$ be arbitrary.

i) If $2 \omega_{0}+\gamma$ (resp. $2 \omega_{1}+\gamma$ ) is the bottom (resp. top) of the essential spectrum, then it can not be an accumulation point of the discrete spectrum of the operator matrix $\mathcal{A}$ provided that

$$
\frac{\lambda}{\omega-\omega_{0}} \in L^{2}\left(\mathbb{R}^{d}\right) \quad\left(\text { resp. } \frac{\lambda}{\omega_{1}-\omega} \in L^{2}\left(\mathbb{R}^{d}\right)\right) .
$$

ii) If $2 \omega_{0}+\gamma$ (resp. $\left.2 \omega_{1}+\gamma\right)$ is an edge but not bottom (resp. top) of the essential spectrum, then it can not be an accumulation point of the discrete spectrum of the operator matrix $\mathcal{A}$ provided that

$$
\frac{\lambda}{\sqrt{\left(\omega-\omega_{0}\right)\left(\omega_{1}-\omega\right)}} \in L^{2}\left(\mathbb{R}^{d}\right)
$$


Proof. i) We give a proof of the claim about the absence of the discrete eigenvalue accumulation at $2 \omega_{1}+\gamma$ from the right. The other claim can be justified analogously. Let the parameters $\alpha$ and $\gamma$ be such that $2 \omega_{1}+\gamma$ is the top of the essential spectrum. Then the second condition in (62) and the Cauchy-Schwarz inequality guarantees that $\Phi\left(\omega_{1}+\gamma\right)$ is finite, see (??). Since $2 \omega_{1}+\gamma$ is the top of the essential spectrum, we have $\Phi\left(\omega_{1}+\gamma\right) \leq 0$. Hence, we easily obtain

$$
\begin{aligned}
\Delta\left(k ; 2 \omega_{1}+\gamma\right) & \leq \Delta\left(k ; 2 \omega_{1}+\gamma\right)-\Phi\left(\omega_{1}+\gamma\right) \\
& =\left(\omega(k)-\omega_{1}\right)\left(1+\alpha^{2} \int \frac{|\lambda(q)|^{2} \mathrm{~d} q}{\left(\omega_{1}-\omega(q)\right)\left(2 \omega_{1}-\omega(k)-\omega(q)\right)}\right),
\end{aligned}
$$

and consequently,

$$
-\Delta\left(k ; 2 \omega_{1}+\gamma\right) \geq \omega_{1}-\omega(k), \quad k \in \mathbb{R}^{d}
$$

By the monotonicity of the function $\Phi$ in the interval $\left(2 \omega_{1}+\gamma, \infty\right)$, we get

$$
\sup _{k \in \mathbb{R}^{d}} \Delta(k ; z)=\Phi\left(z-\omega_{1}\right)<\Phi\left(\omega_{1}+\gamma\right) \leq 0
$$

for all $z \in\left(2 \omega_{1}+\gamma, \infty\right)$. Hence, the multiplication operator $-\Delta(z): L^{2}\left(\mathbb{R}^{d}\right) \rightarrow L^{2}\left(\mathbb{R}^{d}\right)$ is positive for all $z \in\left(2 \omega_{1}+\gamma, \infty\right)$. Since the integral operator $K(z): L^{2}\left(\mathbb{R}^{d}\right) \rightarrow L^{2}\left(\mathbb{R}^{d}\right)$ is well-defined and Hilbert-Schmidt, it follows that the operator

$$
T(z):=(-\Delta(z))^{-1 / 2} K(z)(-\Delta(z))^{-1 / 2}
$$

is also well-defined and Hilbert-Schmidt for all $z \in\left(2 \omega_{1}+\gamma, \infty\right)$.

Next, we define $T\left(2 \omega_{1}+\gamma\right)$ to be the integral operator in $L^{2}\left(\mathbb{R}^{d}\right)$ with the kernel

$$
\Theta\left(k_{1}, k_{2}\right):=\frac{\alpha^{2} \overline{\lambda\left(k_{1}\right)} \lambda\left(k_{2}\right)}{\sqrt{-\Delta\left(k_{1} ; 2 \omega_{1}+\gamma\right)}\left(\omega\left(k_{1}\right)+\omega\left(k_{2}\right)-2 \omega_{1}\right) \sqrt{-\Delta\left(k_{2} ; 2 \omega_{1}+\gamma\right)}} .
$$

Using (65) and the elementary mean-inequality

$$
\frac{1}{2}\left(2 \omega_{1}-\omega\left(k_{1}\right)-\omega\left(k_{2}\right)\right) \geq \sqrt{\left(\omega_{1}-\omega\left(k_{1}\right)\right)\left(\omega_{1}-\omega\left(k_{2}\right)\right)}
$$

we can estimate the kernel in (68) as follows

$$
\left|\Theta\left(k_{1}, k_{2}\right)\right| \leq \frac{\alpha^{2}}{2} \frac{\left|\lambda\left(k_{1}\right)\right|}{\omega_{1}-\omega\left(k_{1}\right)} \frac{\left|\lambda\left(k_{2}\right)\right|}{\omega_{1}-\omega\left(k_{2}\right)}, \quad k_{1}, k_{2} \in \mathbb{R}^{d} .
$$

In view of the second condition in (62), we thus conclude that $\Theta \in L^{2}\left(\mathbb{R}^{d} \times \mathbb{R}^{d}\right)$ with

$$
\|\Theta\|_{L^{2}\left(\mathbb{R}^{d} \times \mathbb{R}^{d}\right)} \leq \frac{\alpha^{2}}{2}\left\|\frac{\lambda}{\omega_{1}-\omega}\right\|_{L^{2}\left(\mathbb{R}^{d}\right)}^{2}<\infty .
$$

Therefore, $T\left(2 \omega_{1}+\gamma\right): L^{2}\left(\mathbb{R}^{d}\right) \rightarrow L^{2}\left(\mathbb{R}^{d}\right)$ is a Hilbert-Schmidt operator and the Lebesgue's dominated convergence theorem guarantees the right-continuity (with respect to the operator norm) of the operator function $T$ at $2 \omega_{1}+\gamma$. This observation and the Weyl inequality (see [11, Chapter IX])

$$
N((-\infty,-1) ; T(z)) \leq N\left((-\infty,-0.5) ; T\left(2 \omega_{1}+\gamma\right)\right)+N\left((-\infty,-0.5) ;-T\left(2 \omega_{1}+\gamma\right)+T(z)\right)
$$

together with the right-continuity (with respect to the operator norm) of the operator functions $\Delta$ and $K$ yield

$$
\begin{aligned}
N\left((-\infty, 0) ;-S\left(2 \omega_{1}+\gamma\right)\right) & =N\left((-\infty, 0) ;-\Delta\left(2 \omega_{1}+\gamma\right)+K\left(2 \omega_{1}+\gamma\right)\right) \\
& =\lim _{z \downarrow 2 \omega_{1}+\gamma} N((-\infty, 0) ;-\Delta(z)+K(z)) \\
& =\lim _{z \downarrow 2 \omega_{1}+\gamma} N((-\infty,-1) ; T(z)) \\
& \leq N\left((-\infty,-0.5) ; T\left(2 \omega_{1}+\gamma\right)\right) .
\end{aligned}
$$


However, $N\left((-\infty,-0.5) ; T\left(2 \omega_{1}+\gamma\right)\right)$ must be a finite number because of the compactness of the operator $T\left(2 \omega_{1}+\gamma\right)$. Now Lemma 2.1 finishes the proof.

ii) Here we give a sketch of the proof of the claim about the absence of the discrete eigenvalue accumulation at $2 \omega_{1}+\gamma$ from the right. The other claim can be justified analogously. Let the parameters $\alpha$ and $\gamma$ be such that $2 \omega_{1}+\gamma$ is an edge but not the top of the essential spectrum. Then, we have $\Phi\left(2 \omega_{1}-\omega_{0}+\gamma\right) \geq 0$ (that is, $\alpha \in\left(0, \alpha_{2}\right]$, see Theorem 2.1). Hence, we easily obtain

$$
\begin{aligned}
\Delta\left(k ; 2 \omega_{1}+\gamma\right) & \geq \Delta\left(k ; 2 \omega_{1}+\gamma\right)-\Phi\left(2 \omega_{1}-\omega_{0}+\gamma\right) \\
& =\left(\omega(k)-\omega_{0}\right)\left(1+\alpha^{2} \int \frac{|\lambda(q)|^{2} \mathrm{~d} q}{\left(2 \omega_{1}-\omega_{0}-\omega(q)\right)\left(2 \omega_{1}-\omega(k)-\omega(q)\right)}\right)
\end{aligned}
$$

and consequently,

$$
\Delta\left(k ; 2 \omega_{1}+\gamma\right) \geq \omega(k)-\omega_{0}, \quad k \in \mathbb{R}^{d} .
$$

By the monotonicity of the function $\Phi$ in the interval $\left(2 \omega_{1}+\gamma, \omega_{0}+F\right)$, we have

$$
\inf _{k \in \mathbb{R}^{d}} \Delta(k ; z)=\Phi\left(z-\omega_{0}\right)>\Phi(F)=0
$$

for all $z \in\left(2 \omega_{1}+\gamma, \omega_{0}+F\right)$. Hence, the multiplication operator $\Delta(z): L^{2}\left(\mathbb{R}^{d}\right) \rightarrow L^{2}\left(\mathbb{R}^{d}\right)$ is positive for all $z \in\left(2 \omega_{1}+\gamma, \omega_{0}+F\right)$. Since the integral operator $K: L^{2}\left(\mathbb{R}^{d}\right) \rightarrow L^{2}\left(\mathbb{R}^{d}\right)$ is well-defined and Hilbert-Schmidt, it follows that the operator

$$
T(z):=(\Delta(z))^{-1 / 2} K(z)(\Delta(z))^{-1 / 2}
$$

is also well-defined and Hilbert-Schmidt for all $z \in\left(2 \omega_{1}+\gamma, \omega_{0}+F\right)$.

Next, we define $T\left(2 \omega_{1}+\gamma\right)$ to be the integral operator in $L^{2}\left(\mathbb{R}^{d}\right)$ with the kernel

$$
\Theta\left(k_{1}, k_{2}\right):=\frac{\alpha^{2} \overline{\lambda\left(k_{1}\right)} \lambda\left(k_{2}\right)}{\sqrt{\Delta\left(k_{1} ; 2 \omega_{1}+\gamma\right)}\left(\omega\left(k_{1}\right)+\omega\left(k_{2}\right)-2 \omega_{1}\right) \sqrt{\Delta\left(k_{2} ; 2 \omega_{1}+\gamma\right)}} .
$$

Using (75) and (69), we get the pointwise estimate

$$
\left|\Theta\left(k_{1}, k_{2}\right)\right| \leq \frac{\alpha^{2}}{2} \frac{\left|\lambda\left(k_{1}\right)\right|}{\sqrt{\left(\omega\left(k_{1}\right)-\omega_{0}\right)\left(\omega_{1}-\omega\left(k_{1}\right)\right)}} \frac{\left|\lambda\left(k_{2}\right)\right|}{\sqrt{\left(\omega\left(k_{2}\right)-\omega_{0}\right)\left(\omega_{1}-\omega\left(k_{2}\right)\right)}} .
$$

It thus follows from the condition (63) that $\Theta \in L^{2}\left(\mathbb{R}^{d} \times \mathbb{R}^{d}\right)$ with

$$
\|\Theta\|_{L^{2}\left(\mathbb{R}^{d} \times \mathbb{R}^{d}\right)} \leq \frac{\alpha^{2}}{2}\left\|\frac{\lambda}{\sqrt{(\omega-m)(M-\omega)}}\right\|_{L^{2}\left(\mathbb{R}^{d}\right)}^{2}<\infty .
$$

Therefore, $T\left(2 \omega_{1}+\gamma\right): L^{2}\left(\mathbb{R}^{d}\right) \rightarrow L^{2}\left(\mathbb{R}^{d}\right)$ is a Hilbert-Schmidt operator and the Lebesgue's dominated convergence theorem guarantees the right-continuity (with respect to the operator norm) of the operator function $T$ at $2 \omega_{1}+\gamma$. The rest of the proof is completely analogous to that of part i) and follows by applying Weyl's inequality together with Lemma 2.1.

Remark 2.2. (i) What we really need in the proof of Theorem 2.4ii) is the square-integrability of

$$
\left(k_{1}, k_{2}\right) \mapsto \frac{\lambda\left(k_{1}\right)}{\sqrt{\omega\left(k_{1}\right)-\omega_{0}}} \frac{1}{\omega\left(k_{1}\right)+\omega\left(k_{2}\right)-2 \omega_{1}} \frac{\lambda\left(k_{2}\right)}{\sqrt{\omega\left(k_{2}\right)-\omega_{0}}}
$$

over $\mathbb{R}^{d} \times \mathbb{R}^{d}$. This requirement is weaker than the condition (63).

(ii) The conditions in (62) are stronger versions of the conditions in (29) and (26). For instance, if the first condition in (62) holds, then the Cauchy-Schwarz inequality yields

$$
\left\|\frac{\lambda}{\sqrt{\omega-\omega_{0}}}\right\|_{L^{2}\left(\mathbb{R}^{d}\right)}^{2} \leq\|\lambda\|_{L^{2}\left(\mathbb{R}^{d}\right)}\left\|\frac{\lambda}{\omega-\omega_{0}}\right\|_{L^{2}\left(\mathbb{R}^{d}\right)}<\infty .
$$

(iii) The way we employ the Weyl inequality in the proofs of Theorems 2.3 and 2.4 is inspired by [33, 26]. 
Corollary 2.1. Let the coupling constant $\alpha>0$ be arbitrary. Assume that both of the following conditions are satisfied

$$
\frac{\lambda}{\omega_{1}-\omega} \in L^{2}\left(\mathbb{R}^{d}\right), \quad \frac{\lambda}{\omega-\omega_{0}} \in L^{2}\left(\mathbb{R}^{d}\right) .
$$

Then the discrete spectrum of the operator matrix $\mathcal{A}$ is finite.

Proof. If (83) holds, then the Cauchy-Schwarz inequality yields

$$
\left\|\frac{\lambda}{\sqrt{\left(\omega_{1}-\omega\right)\left(\omega-\omega_{0}\right)}}\right\|_{L^{2}\left(\mathbb{R}^{d}\right)}^{2} \leq\left\|\frac{\lambda}{\omega_{1}-\omega}\right\|_{L^{2}\left(\mathbb{R}^{d}\right)}\left\|\frac{\lambda}{\omega-\omega_{0}}\right\|_{L^{2}\left(\mathbb{R}^{d}\right)}<\infty .
$$

Hence, Theorems 2.3 and 2.4 imply the finiteness of the discrete spectrum of $\mathcal{A}$ in all cases.

\section{Spectrum of the spin-boson Hamiltonian with two bosons}

Since $\omega$ is bounded, the natural domain of the unperturbed operator $H_{0}$ coincides with $\mathbb{C}^{2} \otimes \mathcal{F}_{s}^{2}$. The first condition in (3) implies the boundedness of the perturbation $H_{\alpha}-H_{0}$ and thus the expression for $H_{\alpha}$ given in (7) generates a self-adjoint operator in the Hilbert space $\mathbb{C}^{2} \otimes \mathcal{F}_{s}^{2}$ with the maximal domain $(c f$. [29. Theorem V.4.3]). For notational convenience, we denote the corresponding self-adjoint operator again by $H_{\alpha}$ and describe its spectrum in the sequel.

We denote by $\mathcal{A}_{\alpha}^{(+)}$and $\mathcal{A}_{\alpha}^{(-)}$the operator matrices of the form (11) corresponding to $\gamma=\varepsilon$ and $\gamma=-\varepsilon$, respectively. As a simple consequence of Theorems $2.1,2.3$ and 2.4 the next result describes the essential spectrum as well as the finiteness of the discrete spectrum of the energy operator $H_{\alpha}$.

Theorem 3.1. Let the coupling constant $\alpha>0$ be arbitrary.

(i) Then the essential spectrum of $H_{\alpha}$ is given by

$$
\sigma_{\mathrm{ess}}\left(H_{\alpha}\right)=\sigma_{\mathrm{ess}}\left(\mathcal{A}_{\alpha}^{(+)}\right) \cup \sigma_{\mathrm{ess}}\left(\mathcal{A}_{\alpha}^{(-)}\right)
$$

with $\sigma_{\mathrm{ess}}\left(\mathcal{A}_{\alpha}^{ \pm}\right)$explicitly determined from Theorem 2.1

(ii) Every edge of the essential spectrum of $H_{\alpha}$ other than $2 \omega_{0} \pm \varepsilon$ and $2 \omega_{1} \pm \varepsilon$ can not be an accumulation point of the discrete spectrum of $H_{\alpha}$.

(iii) If $2 \omega_{0} \pm \varepsilon$ (resp. $2 \omega_{1} \pm \varepsilon$ ) is the bottom (resp. top) of the essential spectrum of $\mathcal{A}_{\alpha}^{( \pm)}$, then it can not be an accumulation point of the discrete spectrum of $H_{\alpha}$ provided that

$$
\frac{\lambda}{\omega-\omega_{0}} \in L^{2}\left(\mathbb{R}^{d}\right) \quad\left(\operatorname{resp} . \frac{\lambda}{\omega_{1}-\omega} \in L^{2}\left(\mathbb{R}^{d}\right)\right)
$$

(iv) If any of $2 \omega_{0} \pm \varepsilon$ (resp. of $2 \omega_{1} \pm \varepsilon$ ) is an edge but not the bottom (resp. top) of the essential spectrum of $\mathcal{A}_{\alpha}^{( \pm)}$, then it can not be an accumulation point of the discrete spectrum of $H_{\alpha}$ provided that

$$
\frac{\lambda}{\sqrt{\left(\omega-\omega_{0}\right)\left(\omega_{1}-\omega\right)}} \in L^{2}\left(\mathbb{R}^{d}\right)
$$

Proof. Consider the unitary transformation $U: \mathbb{C}^{2} \otimes \mathcal{F}_{s}^{2} \rightarrow \mathcal{F}_{s}^{2} \oplus \mathcal{F}_{s}^{2}$, defined by

$$
U:\left(\left(\begin{array}{l}
f_{0}^{(+)} \\
f_{0}^{(-)}
\end{array}\right),\left(\begin{array}{l}
f_{1}^{(+)} \\
f_{1}^{(-)}
\end{array}\right),\left(\begin{array}{l}
f_{2}^{(+)} \\
f_{2}^{(-)}
\end{array}\right)\right) \mapsto\left(\left(\begin{array}{l}
f_{0}^{(+)} \\
f_{1}^{(-)} \\
f_{2}^{(+)}
\end{array}\right),\left(\begin{array}{l}
f_{0}^{(-)} \\
f_{1}^{(+)} \\
f_{2}^{(-)}
\end{array}\right)\right)
$$

By means of this unitary transformation we can block-diagonalize the Hamiltonian $H_{\alpha}$ in (7). In fact, it is not difficult to check that

$$
U^{*} H_{\alpha} U=\operatorname{diag}\left\{P^{*} \mathcal{A}_{\alpha}^{(+)} P, P^{*} \mathcal{A}_{\alpha}^{(-)} P\right\}+\text { operator of rank at most } 4,
$$


where $P: \mathcal{F}_{s}^{2} \rightarrow \mathcal{H}_{1} \oplus \mathcal{H}_{2}$ is the projection operator onto the last two components in the Hilbert space $\mathcal{F}_{s}^{2}$, $\mathcal{A}_{\alpha}^{(+)}$and $\mathcal{A}_{\alpha}^{(-)}$are the operator matrices of the form (11) with $\gamma=\varepsilon$ and $\gamma=-\varepsilon$, respectively. Since the essential spectrum as well as the finiteness of the discrete spectrum of self-adjoint operators are invariant with respect to finite-rank perturbations ( $c f$. [11, Chapter 9]), it follows from (88) that the essential spectrum of $H_{\alpha}$ is given by (84), whereas

$$
\sigma_{\text {disc }}\left(H_{\alpha}\right) \subset \sigma_{\text {disc }}\left(\mathcal{A}_{\alpha}^{(+)}\right) \cup \sigma_{\text {disc }}\left(\mathcal{A}_{\alpha}^{(-)}\right) .
$$

The claims on the discrete spectrum immediately follow from Theorems 2.3 and 2.4 applied for the operator matrices $\mathcal{A}_{\alpha}^{( \pm)}$.

Remark 3.1. (i) It is easy to check that none of $2 \omega_{0} \pm \varepsilon$ can ever be the bottom of the essential spectrum of $H_{\alpha}$. Hence, discrete eigenvalues of $H_{\alpha}$ can never accumulate to the bottom of the essential spectrum of $H_{\alpha}$ from the left. The top of the essential spectrum of $H_{\alpha}$ can never be $2 \omega_{1} \pm \varepsilon$ whenever (29) is not satisfied. Consequently, discrete eigenvalues of $H_{\alpha}$ can never accumulate to the top of the essential spectrum of $H_{\alpha}$ from the right whenever (29) is violated.

(ii) It follows from Corollary 2.1 that the discrete spectrum of $H_{\alpha}$ is always finite whenever the infrared regularity conditions (85) are satisfied. We believe that, at least for certain values of the spatial dimension $d \geq 1$, conditions of type (85)- (86) are necessary to guarantee the finiteness of the discrete spectrum near the corresponding edges of the essential spectrum. In fact, the violation of a infrared regularity condition is equivalent to the fact that the corresponding edge of the essential spectrum of the two-boson system is a resonance state and in this case we expect an effect analogous to the Efimov effect from the spectral theory of the standard three-body Schrödinger operators, $c f$. [28, 45, 48, 4].

\section{Acknowledgments}

Most of the results in this paper were obtained during my stay at University College London (UCL). I am grateful to Prof. A. V. Sobolev for fruitful discussions and I thank the Department of Mathematics at UCL for the kind hospitality. I would also like to thank Prof. H. Spohn for stimulating personal communications on the subject of this paper. The financial support of the Swiss National Science Foundation through the Early Postdoc.Mobility grant No. 168723 is gratefully acknowledged.

\section{References}

[1] Abdesselam, A. The ground state energy of the massless spin-boson model. Ann. Henri Poincaré 12, 7 (2011), $1321-1347$.

[2] Abdullaev, Z. I., And Ikromov, I. A. Finiteness of the number of eigenvalues of the two-particle Schrödinger operator on a lattice. Teoret. Mat. Fiz. 152, 3 (2007), 502-517.

[3] Adamyan, V., Mennicken, R., and Saurer, J. On the discrete spectrum of some selfadjoint operator matrices. J. Operator Theory 39, 1 (1998), 3-41.

[4] Albeverio, S., Lakaev, S. N., and Muminov, Z. I. Schrödinger operators on lattices. The Efimov effect and discrete spectrum asymptotics. Ann. Henri Poincaré 5, 4 (2004), 743-772.

[5] Angelescu, N., Minlos, R. A., Ruiz, J., And Zagrebnov, V. A. Lower spectral branches of a spin-boson model. J. Math. Phys. 49, 10 (2008), 102105, 29.

[6] Arai, A. Essential spectrum of a self-adjoint operator on an abstract Hilbert space of Fock type and applications to quantum field Hamiltonians. J. Math. Anal. Appl. 246, 1 (2000), 189-216.

[7] Arai, A., and Hirokawa, M. On the existence and uniqueness of ground states of a generalized spin-boson model. J. Funct. Anal. 151, 2 (1997), 455-503.

[8] Atkinson, F. V., Langer, H., Mennicken, R., and Shkalikov, A. A. The essential spectrum of some matrix operators. Math. Nachr. $16^{r} 7$ (1994), 5-20.

[9] Bach, V., Ballesteros, M., Könenberg, M., and Menrath, L. Existence of ground state eigenvalues for the spin-boson model with critical infrared divergence and multiscale analysis. J. Math. Anal. Appl. 453, 2 (2017), 773-797.

[10] BaCh, V., Fröhlich, J., And Sigal, I. M. Quantum electrodynamics of confined nonrelativistic particles. Adv. Math. 137, 2 (1998), 299-395. 
[11] Birman, M. S., And Solomjak, M. Z. Spectral theory of selfadjoint operators in Hilbert space. Mathematics and its Applications (Soviet Series). D. Reidel Publishing Co., Dordrecht, 1987. Translated from the 1980 Russian original by S. Khrushchëv and V. Peller.

[12] Boutet de Monvel, A., And Sahbani, J. On the spectral properties of the spin-boson Hamiltonians. Lett. Math. Phys. 44, 1 (1998), 23-33.

[13] Bräunlich, G., Hasler, D., and Lange, M. On Asymptotic Expansions in Spin-Boson Models. Ann. Henri Poincaré 19, 2 (2018), 515-564.

[14] Davies, E. B. Symmetry breaking for molecular open systems. Ann. Inst. H. Poincaré Sect. A (N.S.) 35, 2 (1981), 149-171.

[15] De Roeck, W., Griesemer, M., and Kupiainen, A. Asymptotic completeness for the massless spin-boson model. Adv. Math. 268 (2015), 62-84.

[16] Dereziński, J., And Jakšić, V. Spectral theory of Pauli-Fierz operators. J. Funct. Anal. 180, 2 (2001), 243-327.

[17] Duclos, P., And Exner, P. Curvature-induced bound states in quantum waveguides in two and three dimensions. Rev. Math. Phys. 7, 1 (1995), 73-102.

[18] Fröhlich, H. Electrons in lattice fields. Adv. in Phys. 3 (1954), 325-362.

[19] Galtbayar, A., Jensen, A., and Yajima, K. The Nelson model with less than two photons. Ann. Henri Poincaré 4, 2 (2003), 239-273.

[20] Gérard, C. Asymptotic completeness for the spin-boson model with a particle number cutoff. Rev. Math. Phys. 8, 4 (1996), 549-589.

[21] Hasler, D., And Herbst, I. Ground states in the spin boson model. Ann. Henri Poincaré 12, 4 (2011), 621-677.

[22] Hirokawa, M. Remarks on the ground state energy of the spin-boson model. An application of the WignerWeisskopf model. Rev. Math. Phys. 13, 2 (2001), 221-251.

[23] Hübner, M., And Spohn, H. Spectral properties of the spin-boson Hamiltonian. Ann. Inst. H. Poincaré Phys. Théor. 62, 3 (1995), 289-323.

[24] Ibrogimov, O. O. On the spectrum of a tridiagonal operator matrix and its application to radioactive decay. Preprint, Dec. 2018.

[25] Ibrogimov, O. O. Spectral Analysis of the Spin-Boson Hamiltonian with Two Photons for Arbitrary Coupling. Ann. Henri Poincaré 19, 11 (2018), 3561-3579.

[26] Ibrogimov, O. O., And Tretter, C. On the spectrum of an operator in truncated Fock space. Operator Theory: Advances and Applications 263 (2018), 321-334.

[27] Ikromov, I. A., ANd Sharipov, F. On the discrete spectrum of the nonanalytic matrix-valued Friedrichs model. Funktsional. Anal. i Prilozhen. 32, 1 (1998), 63-65.

[28] Jafaev, D. R. On the theory of the discrete spectrum of the three-particle Schrödinger operator. Mat. Sb. (N.S.) 94(136) (1974), 567-593, 655-656.

[29] Kato, T. Perturbation theory for linear operators. Springer-Verlag, Berlin, 1995. Reprint of the 1980 edition.

[30] Klaus, M. On the bound state of Schrödinger operators in one dimension. Ann. Physics 108, 2 (1977), 288-300.

[31] Könenberg, M., Merkli, M., and Song, H. Ergodicity of the spin-boson model for arbitrary coupling strength. Comm. Math. Phys. 336, 1 (2015), 261-285.

[32] Kraus, M., Langer, M., And Tretter, C. Variational principles and eigenvalue estimates for unbounded block operator matrices and applications. J. Comput. Appl. Math. 171, 1-2 (2004), 311-334.

[33] Lakaev, S. N., And Muminov, M. E. Essential and discrete spectrum of the three-particle Schrödinger operator on a lattice. Teoret. Mat. Fiz. 135, 3 (2003), 478-503.

[34] Langer, H., LAnger, M., And Tretter, C. Variational principles for eigenvalues of block operator matrices. Indiana University Mathematics Journal 51, 6 (2002), 1427-1460.

[35] Marletta, M., and Tretter, C. Essential spectra of coupled systems of differential equations and applications in hydrodynamics. J. Differential Equations 243, 1 (2007), 36-69.

[36] Minlos, R., And Spohn, H. The three-body problem in radioactive decay: the case of one atom and at most two photons. In Topics in statistical and theoretical physics, vol. 177 of Amer. Math. Soc. Transl. Ser. 2. Amer. Math. Soc., Providence, RI, 1996, pp. 159-193.

[37] Miyao, T. Polaron with at most one phonon in the weak coupling limit. Monatsh. Math. 157, 4 (2009), 365-378. 
[38] Møller, J. S. The translation invariant massive Nelson model. I. The bottom of the spectrum. Ann. Henri Poincaré 6, 6 (2005), 1091-1135.

[39] Møller, J. S., And Rasmussen, M. G. The translation invariant massive Nelson model: II. The continuous spectrum below the two-boson threshold. Ann. Henri Poincaré 14, 4 (2013), 793-852.

[40] Muminov, M., Neidhardt, H., and Rasulov, T. On the spectrum of the lattice spin-boson Hamiltonian for any coupling: 1D case. J. Math. Phys. 56, 5 (2015), 053507, 24.

[41] Newton, R. G. Bounds on the number of bound states for the Schrödinger equation in one and two dimensions. J. Operator Theory 10, 1 (1983), 119-125.

[42] Rasulov, T. K. On branches of the essential spectrum of the lattice spin-boson model with at most two photons. Teoret. Mat. Fiz. 186, 2 (2016), 293-310.

[43] Setô, N. Bargmann's inequalities in spaces of arbitrary dimension. Publ. Res. Inst. Math. Sci. 9 (1973/74), 429-461.

[44] Skibsted, E. Spectral analysis of N-body systems coupled to a bosonic field. Rev. Math. Phys. 10, 7 (1998), 989-1026.

[45] Sobolev, A. V. The Efimov effect. Discrete spectrum asymptotics. Comm. Math. Phys. 156, 1 (1993), $101-126$.

[46] Spohn, H. The polaron at large total momentum. J. Phys. A 21, 5 (1988), 1199-1211.

[47] Spohn, H. Ground state(s) of the spin-boson Hamiltonian. Comm. Math. Phys. 123, 2 (1989), $277-304$.

[48] Tamura, H. The Efimov effect of three-body Schrödinger operators. J. Funct. Anal. 95, 2 (1991), $433-459$.

[49] Tretter, C. Spectral theory of block operator matrices and applications. Imperial College Press, London, 2008.

[50] Zhukov, Y. V., And Minlos, R. A. The spectrum and scattering in the "spin-boson" model with at most three photons. Teoret. Mat. Fiz. 103, no. 1 (1995), 63-81. 